\title{
A STUDY ON PATTERN OF PRESCRIBING MEDICATIONS USED IN SECONDARY PREVENTION OF STROKE
}

\section{JITHIN KC, ARYA G, LEKSHMI P NAIR, LAKSHMI R*}

Department of Pharmacy Practice, Amrita School of Pharmacy, Amrita Vishwa Vidyapeetham, Amrita University, Kochi, Kerala, India. Email: lakshmir@aims.amrita.edu

Received: 15 March 2016, Revised and Accepted: 05 September 2016

\section{ABSTRACT}

Objectives: The main objectives of this study were to estimate the demographic details of patients with the first incidence of stroke and to study the various risk factors for ischemic stroke.

Methods: It was a retrospective, observational study carried out for 1 year at the Department of Stroke Medicine of a tertiary care teaching hospital in Kerala, India. A total of 636 patients were admitted under stroke medicine during the period from July 1, 2014, to July 1, 2015, and who satisfied the inclusion and exclusion criteria were included in the study. A standardized data collection form was prepared and necessary data were collected from patient's medical records.

Results: The maximum number of patients was in the age group of 60-69 years. $65.1 \%$ patients were residing in the rural area. $60.7 \%$ patients were male. Most of them had hypertension as common comorbidity (21.35\%) and the highest number of patients had diabetes mellitus and hypertension (21.7\%). Majority of the patients were prescribed with antiplatelets (94.91\%), followed by statins (93.8\%).

Conclusion: A strict control on the comorbid conditions and sticking on to the medications can prevent the occurrence of future stroke.

Keywords: Stroke, Secondary prevention, Drugs, Prescribing pattern.

(C) 2016 The Authors. Published by Innovare Academic Sciences Pvt Ltd. This is an open access article under the CC BY license (http://creativecommons. org/licenses/by/4. 0/) DOI: http://dx.doi.org/10.22159/ajpcr.2016.v9s3.14071

\section{INTRODUCTION}

Stroke is a preventable and treatable disease. Over the last two decades, an increasing picture of evidence has rewritten the traditional belief that reason behind stroke was merely aging which caused disability and death. Evidence shows effective primary and secondary prevention strategies, categorizing high-risk patients, and also other interventions done soon after the onset of symptoms have given a fruitful outcome in the management of the same [1]. The various types of stroke are as follows:

- Ischemic stroke

- Hemorrhagic stroke

- Transient ischemic attack (TIA).

Stroke is classically characterized as a neurological deficit attributed to an acute focal injury of the central nervous system by a vascular cause, including cerebral infarction, intracerebral hemorrhage, intraventricular hemorrhage, and subarachnoid hemorrhage or infarction caused by infection or tumor with symptoms lasting more than $24 \mathrm{hrs}$ or leading to death [2]. The ultimate result of ischemic cascade initiated by acute stroke is neuronal death along with an irreversible loss of neuronal function [3]. TIAs are often referred to as ministrokes, warning strokes, or transient strokes $[4,5]$.

\section{Symptoms [6]}

- Sudden numbness or weakness of the face, arm, or leg, especially on one side of the body.

- Sudden confusion, trouble speaking, or understanding.

- Sudden trouble seeing in one eye or both.

- Sudden trouble walking, dizziness, loss of balance, or coordination.

- Sudden severe headache with no known causes.

Risk factors for stroke [7]

Non-modifiable risk factors: Age, sex, low birth weight, race, family history of stroke, or TIA.
Modifiable risk factors: Cigarette smoking, hypertension, high tota cholesterol, low HDL cholesterol, atrial fibrillation, obesity, physical inactivity, diet, asymptomatic carotid stenosis, postmenopausal hormone therapy, high sodium/low potassium diet, cardiovascular disorders, alcohol, etc.

\section{Pharmacotherapy}

Antiplatelet therapy is the gold standard for the secondary prevention of non-cardioembolic strokes and anticoagulants for cardioembolic one. All antihypertensive drugs are used for the management of elevated blood pressure. ACE inhibitor + diuretic are used for both previously hypertensive or previously normotensive. Statins are initiated for both dyslipidemics and also for normal lipids [6].

\section{METHODS}

\section{Study design}

This was a retrospective, observational study.

\section{Study duration}

The study period was from July 1, 2015, to July 1, 2016.

\section{Study population}

Patients with first incidence of ischemic stroke admitted to the Department of Stroke Medicine of a tertiary care teaching hospital in Kerala during the period (July 1, 2014, to July 1,2015) and who satisfied the inclusion and exclusion criteria were included in the study.

\section{Inclusion criteria}

- Patients who had the first incidence of ischemic stroke.

- Patients who were discharged with drugs for secondary prevention

- Patients of age group $\geq 18$ years.

Exclusion criteria

- Patients diagnosed with hemorrhagic stroke.

- Patients diagnosed with cancer and renal failure. 
Sample size

Sample size (n) was calculated statistically on the basis of the first incidence of stroke and patients prescribed with secondary prevention medications. The sample size was $\mathrm{n}>237$.

\section{RESULTS}

In this study, a total of 295 patients were studied during a period of 12 months. The age range was from 18 years to 100 years, with a mean age of 64.5 years. The maximum number of patients, i.e., $78(26.44 \%)$ were in the age group of $60-69$ years and $67(22.71 \%)$ were in the age group of 70-79 years (Table 1). Furthermore, majority of them were residing in rural area $(65.1 \%)$ (Table 2$)$.

Out of 295 patients, $60.7 \%$ were male and 39.3\% were female (Fig. 1). After the assessment of comorbidities, the most common one was hypertension. Highest number was hypertension combined with diabetes mellitus (DM) in 64 patients (21.7\%), followed by hypertension alone in 63 patients (21.35\%), hypertension combined with dyslipidemia and DM in 51 patients (51\%), and cardiac disease in 49 patients (16.6\%) (Table 3).

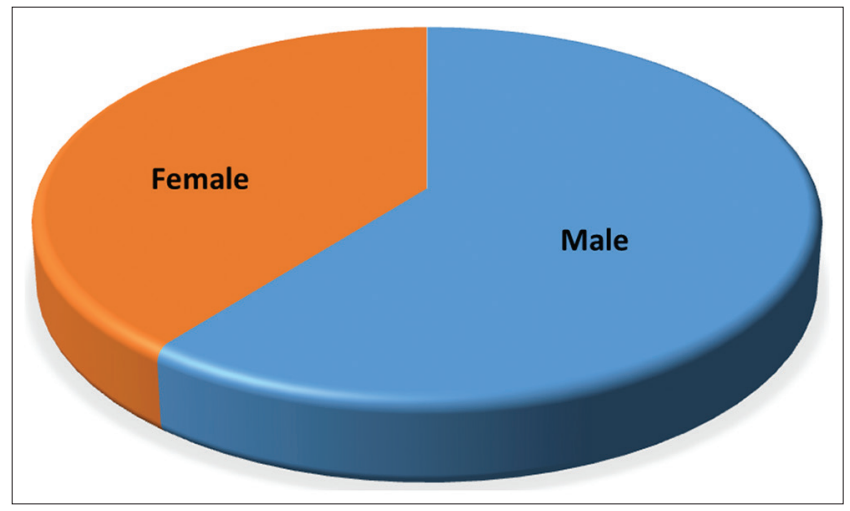

Fig. 1: Gender distribution of patients

Table 1: Age distribution of patients

\begin{tabular}{ll}
\hline Age (years) & Number of patients $\mathbf{n = 2 9 5}(\mathbf{\%})$ \\
\hline $18-29$ & $7(2.4)$ \\
$30-39$ & $8(2.71)$ \\
$40-49$ & $40(13.55)$ \\
$50-59$ & $69(23.38)$ \\
$60-69$ & $78(26.44)$ \\
$70-79$ & $67(22.71)$ \\
$\geq 80$ & $26(8.81)$ \\
\hline
\end{tabular}

Table 2: Residential categorization of patients

\begin{tabular}{ll}
\hline Area of residence & Number of patients $\mathbf{n}=\mathbf{2 9 5}(\%)$ \\
\hline Rural & $192(65.1)$ \\
Urban & $103(34.9)$ \\
\hline
\end{tabular}

Table 3: Distribution of co-morbidities in study sample

\begin{tabular}{ll}
\hline Co-morbidities & Number of patients $\mathbf{n = 2 9 5}(\mathbf{\%})$ \\
\hline HTN & $63(21.35)$ \\
DM & $24(8.13)$ \\
DLP & $11(3.72)$ \\
Cardiac disease & $49(16.6)$ \\
HTN+DM & $64(21.7)$ \\
HTN+DLP & $23(7.8)$ \\
HTN+DM+cardiac disease & $27(9.1)$ \\
HTN+DLP+DM & $51(17.3)$ \\
HTN+cardiac disease & $8(2.7)$ \\
\hline
\end{tabular}

Among 295 patients, antiplatelet drug such as aspirin was administered to $55(18.6 \%)$ patients, clopidogrel was administered to $40(14.23 \%)$ patients, and aspirin-clopidogrel combination was given to 185 (62.7\%) patients (Table 4). Cardioembolic stroke patients were treated with anticoagulants such as warfarin to $26(8.8 \%)$ patients and heparin to $9(3 \%)$ patients (Table 5).

Patients with hypertension were treated with different classes of antihypertensives such as calcium channel blockers to 161 (54.5\%) patients, followed by angiotensin receptor blockers for $86(29.1 \%)$ patients and beta-blockers to $72(24.4 \%)$ patients (Table 6). Atorvastatin was given to $268(90.8 \%)$ patients for aggressive lipid reduction (Table 7). For diabetic control, insulin was prescribed to 38 (12.8\%) patients and metformin to $30(10.1 \%)$ patients (Table 8); however, in combination therapy, metformin had the highest percentage $(27.1 \%)$ than insulin (19.3\%).

Antiepileptics such as levetiracetam were given to $23(7.7 \%)$ patients, followed by clonazepam to $20(6.7 \%)$ patients to control post-stroke seizures (Table 9). Post-stroke depression was managed using antidepressants such as fluoxetine $(9.1 \%)$ and venlafaxine (3.7\%). Vitamin supplements were given to 118 patients to decrease the hyperhomocysteinemia and possible neural damage; Remylin D (folic acid, B12, and B6) was the most prescribed one. Lactulose was given for constipation in 100 patients and gastrointestinal (GI) protectives were given to minimize the antiplatelet-induced gastric irritation and ulceration and the most commonly prescribed one was pantoprazole to 164 patients.

\section{DISCUSSION}

The male to female ratio of the study patients was 1.54:1 and male were more in number; further, the most common age group experienced stroke was between 60-69 years. This result closely relates with studies done by Bhattacharya et al. [8] and Sethi [9]. Utmost of the patients who were included in this study were staying in the rural area, i.e., 179 (60.7\%), and

Table 4: Prescription pattern of antiplatelets in study sample

\begin{tabular}{ll}
\hline Antiplatelets & Number of patients $\mathbf{n}=\mathbf{2 9 5}(\%)$ \\
\hline Clopidogrel & $40(14.23)$ \\
Aspirin & $55(18.6)$ \\
Clopidogrel+aspirin & $185(62.7)$ \\
\hline
\end{tabular}

Table 5: Prescription pattern of anticoagulants in study sample

\begin{tabular}{ll}
\hline Anticoagulants & Number of patients $\mathbf{n = 2 9 5}$ (\%) \\
\hline Warfarin & $26(8.8)$ \\
Heparin & $9(3)$ \\
Dabigatran & $5(1.6)$ \\
\hline
\end{tabular}

Table 6: Prescription pattern of anti-hypertensives

\begin{tabular}{ll}
\hline Antihypertensives & Number of patients $\mathbf{n = 2 9 5}(\%)$ \\
\hline CCB & $161(54.5)$ \\
ACE inhibitor & $13(4.4)$ \\
ARB & $86(29.1)$ \\
Diuretics & $33(11.1)$ \\
Beta blocker & $72(24.4)$ \\
Alpha blocker & $18(6.1)$ \\
CCB+Beta blocker & $1(0.33)$ \\
\hline
\end{tabular}

Table 7: Prescription pattern of statins in the study sample

\begin{tabular}{ll}
\hline Lipid lowering agents & Number of patients $\mathbf{n}=\mathbf{2 9 5}(\%)$ \\
\hline Atorvastatin & $268(90.8)$ \\
Rosuvastatin & $9(3)$ \\
\hline
\end{tabular}


Table 8: Prescription pattern of antidiabetics in the study sample

\begin{tabular}{ll}
\hline Antidiabetics & Number of patients $\mathbf{n = 2 9 5}(\mathbf{0})$ \\
\hline Insulin & $38(12.8)$ \\
Metformin & $30(10.0)$ \\
Glimipride & $14(4.74)$ \\
Glibenclamide & $2(0.67)$ \\
Glipizide & $2(0.67)$ \\
Metformin+insulin & $17(5.7)$ \\
Metformin+glimipride & $28(9.49)$ \\
Metformin+sitagliptin & $4(1.35)$ \\
Metformin+glibenclamide & $1(0.33)$ \\
Glimipride+insulin & $2(0.67)$ \\
\hline
\end{tabular}

Table 9: Prescription pattern of antiepileptics

\begin{tabular}{ll}
\hline Anticonvulsants & Number of patients $\mathbf{n = 2 9 5}(\mathbf{\%})$ \\
\hline Clonazepam & $20(6.7)$ \\
Levitiracetam & $23(7.7)$ \\
Phenytoin & $13(4.4)$ \\
Gabapentein & $3(1)$ \\
Sodium valproate & $4(1.35)$ \\
\hline
\end{tabular}

116 (39.3\%) in urban area. The present study emphasized the risk factors and drugs used in secondary prevention of stroke. In our study patients, the most common risk factors associated with stroke were hypertension and the highest was hypertension combined with DM, followed by cardiac diseases. In the present study, most of the patients were prescribed with antiplatelets, anticoagulants, and statins, which are in close quarter with the American Heart Association Guidelines. The most frequently prescribed drug in our study was atorvastatin (90.8\%), followed by aspirin-clopidogrel combination (62.7\%) and calcium channel blockers (54.5\%). Multivitamins were also prescribed extensively to 118 patients to control hyperhomocysteinemia and neural damage. Levetiracetam was prescribed to control post-stroke seizures which showed an even result as that of a study done by Belcastro et al. [10]. Lactulose was prescribed to manage constipation and GI protectants such as pantoprazole were used to minimize gastric bleeding.

\section{CONCLUSION}

The present study helped identify copious risk factors and prerequisite for early and satisfactory management of stroke to prevent future complications. Majority of patients in this study were male $(60.7 \%)$ between the age group 60-69 years living in rural area (65.1\%). Residential categorization revealed that patients were more from rural area rather from urban. Common comorbidities were identified and in which hypertension. Diabetes and dyslipidemia were high in number. These were the modifiable risk factors which could be controlled with adequate treatment and medications. The information from the study could be helpful in avoidance of risk factors and early apposite medical therapy in these patients. The study also revealed various medicines prescribed in stroke such as antiplatelets, anticoagulant, antihypertensive, statins, and antidiabetics. Post-stroke disabilities such as post-stroke depression, post-stroke seizures, neural damage, and constipation were minimized by prescribing drugs such as antidepressants, antiepileptics, multivitamins, and laxatives, respectively. GI protectants were additionally added to reduce gastric bleeding.

A first-ever stroke significantly increases the likelihood of further events; thus, secondary prevention is of major importance. The absolute and relative risks of recurrent stroke are highest early after the first stroke but remain elevated for several years thereafter. Effort on the secondary prevention should be initiated as soon as possible and continued for several years to gain the greatest benefit [11]. Thus, it is imperative that public should be made aware of the significance for the secondary prevention of stroke as it helps to reduce the recurrence.

\section{REFERENCES}

1. National Collaborating Centre for Chronic Conditions. Stroke: National Clinical Guideline for Diagnosis and Initial Management of Acute Stroke and Transient Ischaemic Attack (TIA). $3^{\text {rd }}$ ed. London: Royal College of Physicians; 2008.

2. Sacco RL, Kasner SE, Broderick JP, Caplan LR, Connors JJ, Culebras A, et al. An updated definition of stroke for the $21^{\text {st }}$ century: A statement for healthcare professionals from the American Heart Association/ American Stroke Association. Stroke 2013;44(7):2064-89.

3. Deb P, Sharma S, Hassan KM. Pathophysiologic mechanisms of acute ischemic stroke: An overview with emphasis on therapeutic significance beyond thrombolysis. Pathophysiology 2010;17(3):197-218.

4. Johnston SC. Clinical practice. Transient ischemic attack. N Engl J Med 2002;347(21):1687-92.

5. Albers GW, Caplan LR, Easton JD, Fayad PB, Mohr JP, Saver JL, et al. Transient ischemic attack - Proposal for a new definition. N Engl J Med 2002;347(21):1713-6.

6. Dipiro JT, Talbert RL, Yee GC, Matzke GR, Wells BG, Posey LM Pharmacotherapy a Pathophysiologic Approach. $8^{\text {th }}$ ed. New York: McGraw-Hill Medical; 2008.

7. Lakshmi R, James E, Kirthivasan R. Study on impact of clinical pharmacist's interventions in the optimal use of oral anticoagulants in stroke patients. Indian J Pharm Sci 2013;75(1):53-9.

8. Bhattacharya S, Saha SP, Basu A, Das SK. A 5 years prospective study of incidence, morbidity and mortality profile of stroke in a rural community of eastern India. J Indian Med Assoc 2005;103(12):655-9.

9. Sethi PK. Stroke-incidence in India and management of ischaemic stroke. Neuroscience 2002;4(3):139-41.

10. Belcastro V, Costa C, Galletti F, Autuori A, Pierguidi L, Pisani F, et al. Levetiracetam in newly diagnosed late-onset post-stroke seizures: A prospective observational study. Epilepsy Res 2008;82(2-3):223-6.

11. Burn J, Dennis M, Bamford J, Sandercock P, Wade D, Warlow C. Longterm risk of recurrent stroke after a first-ever stroke. The Oxfordshire Community Stroke Project. Stroke 1994;25(2):333-7. 